\title{
Metaciclogénesis: un proceso fundamental en la biología de Leishmania
}

\author{
Carlos E. Muskus, Marcel Marín Villa \\ Programa de Estudio y Control de Enfermedades Tropicales, PECET, \\ Universidad de Antioquia, Medellín, Colombia.
}

La metaciclogénesis es un proceso que experimentan naturalmente los promastigotes de Leishmania en el tracto digestivo del insecto vector y cuya finalidad es transformar los promastigotes en formas altamente infectivas y capaces de sobrevivir en el hospedero vertebrado, donde es sometido a los ataques por parte del sistema inmune. Se ha demostrado que este fenómeno ocurre también en promastigotes en crecimiento en cultivos axénicos in vitro. El proceso de metaciclogénesis se asoció inicialmente con cambios morfológicos, observándose que los promastigotes cambiaban su forma y tamaño con incremento en la longitud del flagelo. Luego, se logró asociar con este fenómeno la expresión de ciertas moléculas implicadas en virulencia, como el lipofosfoglicano (LPG) y una proteasa de superficie, la gp63. Se demostró que estas moléculas experimentaban cambios tanto cuantitativos como cualitativos a medida que los promastigotes se diferencian de promastigotes procíclicos no infectivos a metacíclicos o infectivos. Hoy en día, mediante técnicas de hibridización substractiva con cADN o de amplificación diferencial, se han logrado identificar genes cuyo patrón de expresión está íntimamente ligado al proceso de metaciclogénesis. Se han identificado moléculas como el producto del gen B y una proteína asociada con la metaciclogénesis (Mat-1), las cuales se expresan exclusivamente en promastigotes metacíclicos, mientras que otras como las proteínas Meta-1, SHERP y HASP se sobrexpresan en los promastigotes metacíclicos. Sin embargo, la función y asociación de estas proteínas con este patrón particular de expresión y virulencia se está empezando a evaluar.

Palabras clave: metaciclogénesis, Leishmania, promastigotes, LPG, gp63.

\section{Metacyclogenesis: a basic process in Leishmania biology}

Metacyclogenesis is a process whereby Leishmania transforms from poorly infective procyclic promastigotes into highly infective metacyclic promastigotes. In nature, metacyclogenesis occurs in the insect vector. This transformation is accompanied by an increased ability to infect and survive in the vertebrate host, where the parasite is attacked by the host's immune system. Metacyclogenesis has also been shown to occur in axenic cultures of promastigotes. Morphological changes in size and shape, and length of flagellum were first associated with differentiation in the insect gut and in different phases of growth in culture. Later, the expression of molecules such as LPG and the surface protease gp63 were associated with this process. These two molecules were observed to undergo qualitative and quantitative modifications as the promastigotes differentiated from procyclic to metacyclic forms. Using cDNA subtractive hybridization-based methods or differential amplification, previously unknown genes tightly linked to metacyclogenesis have been identified. Gene products exclusively expresed in metacyclic promastigotes included a gene B product and Mat-1 -a gene associated with metacyclogenesis. Other proteins, Meta-1, SHERP and HASP, were up-regulated during the metacyclic stage. The function and stage-regulated expression of these molecules and their relationship with infectivity are now under investigation.

Key words: metacyclogenesis, Leishmania, promastigotes, LPG, gp63 
El parásito Leishmania es un protozoo que pertenece a la familia Trypanosomatidae, orden Kinetoplastida y género Leishmania. Es transmitido por vectores flebotomíneos, clasificados dentro del género Phlebotomus en el Viejo Mundo y Lutzomyia en el Nuevo Mundo. Su ciclo de vida, además del hombre, involucra reservorios vertebrados como el perro (Canis familiaris), oso perezoso (Choloepus spp. y Bradypus spp.), zarigüeya (Didelphis marsupialis) y algunos roedores, entre otros. Las especies de parásitos pertenecientes al género Leishmania se agrupan en dos subgéneros de acuerdo con el sitio donde se desarrollan en el insecto vector. En el subgénero Leishmania se incluyen las especies que se desarrollan en el intestino medio de los flebotomíneos, como las especies del Viejo Mundo y el complejo mexicana del Nuevo Mundo, mientras que en el subgénero Viannia se agrupan las especies que se desarrollan en el intestino posterior a la altura del triángulo pilórico, dentro del cual se incluyen el resto de las especies del Nuevo Mundo, como Leishmania panamensis, Leishmania braziliensis y Leishmania guyanensis (1).

Los parásitos de Leishmania presentan dos estadios en su ciclo de vida: el promastigote, la forma flagelada, móvil, que se encuentra en los insectos vectores, y el amastigote, que no presenta flagelo, es inmóvil y se encuentra dentro de los macrófagos del hospedero mamífero. En estos hospederos, el parásito debe ser capaz de adaptarse a vivir en tres microambientes diferentes: 1) en el tracto digestivo del insecto vector; 2) en el compartimiento extracelular de los hospederos mamíferos, una vez que el promastigote es inoculado por el insecto vector, y 3 ) en los fagolisosomas de los macrófagos $(1,2)$.

Durante el desarrollo del parásito en el tracto digestivo del vector se han descrito varias formas morfológicas (3). Estos cambios morfológicos, los cuales se correlacionan con el desarrollo del

$\overline{\text { Correspondencia: }}$

Carlos E. Muskus, PECET, Universidad de Antioquia, Medellín, Colombia

carmusk@yahoo.com

Recibido: 20/09/01; aceptado: 16/05/02 parásito, se ha encontrado que están asociados con cambios genéticos y bioquímicos $(4,5)$, los cuales, en algunos casos, modulan funciones biológicas.

Esta serie de cambios que conllevan la transformación de promastigotes no infectivos o poco infectivos (procíclicos) en promastigotes infectivos (metacíclicos) en el insecto vector y que les permite sobrevivir en ese nuevo ambiente dentro del hospedero mamífero es lo que se conoce como metaciclogénesis (6). Se ha demostrado que el proceso de metaciclogénesis ocurre también en cultivos axénicos en varias especies de Leishmania (6).

En esta revisión se presentan algunas de las evidencias experimentales que demuestran la existencia de promastigotes metacíclicos o infectivos y cómo este incremento en la infectividad se relaciona con la expresión de nuevas moléculas o la sobrexpresión de otras a medida que el parásito se transforma de promastigote procíclico o no infectivo a promastigote metacíclico. Además, se describen algunas de las implicaciones de este proceso de metaciclogénesis en el ciclo de vida de Leishmania.

\section{Evidencias de un estadio infectivo en promastigotes de Leishmania}

Cuando crecen en medios de cultivo in vitro o en el insecto vector, los parásitos de Leishmania presentan dos fases de crecimiento: una fase replicativa, en la cual el número de parásitos se incrementa, conocida como fase logarítmica, y otra fase donde el número de promastigotes permanece casi constante, conocida como fase estacionaria $(6,7)$. En 1984, Sacks y Perkins (7), empleando macrófagos peritoneales murinos, informaron que la infectividad de promastigotes de Leishmania major obtenidos en fase estacionaria era mayor que la de promastigotes obtenidos en la fase logarítmica de crecimiento. La mayor infectividad observada con promastigotes de fase estacionaria había sido observada anteriormente en hámsters por Giannini con una cepa de Leishmania donovani (11). Para determinar si la transformación hacia un estadio más infectivo ocurría durante el 
crecimiento del parásito en el vector, se obtuvieron promastigotes del intestino de Lutzomya anthophora durante los primeros días de infección, se inocularon en ratones susceptibles BALB/c y se observó que éstos eran avirulentos. En contraste, los promastigotes del intestino del mismo vector obtenidos entre los días 7 y 10 fueron altamente virulentos (8).

Inicialmente, los cambios cualitativos y cuantitativos en la composición de carbohidratos se demostraron por aglutinación con lectinas a medida que los parásitos se diferenciaban de fase logarítmica a fase estacionaria de crecimiento. En experimentos de aglutinación con varios clones de L. major y la aglutinina de maní (PNA), se observó un $100 \%$ de aglutinación de los promastigotes en fase logarítmica, mientras que sólo se observó aglutinación en un 10\% de los promastigotes de fase estacionaria (9). Ensayos realizados por Da Silva y Sacks (10) mostraron que los parásitos en fase logarítmica y aglutinados en su totalidad con PNA eran muy poco infectivos en ratones BALB/c, en contraste con la población de promastigotes que aglutinó en un $10 \%$ y que fue un poco más infectiva. El enriquecimiento de la población de promastigotes PNA negativos de fase estacionaria mostró una virulencia incrementada (10). Lo anterior sugiere que aunque no se observen cambios morfológicos evidentes en los promastigotes de fase estacionaria, algunos marcadores de metaciclogénesis, como el grado de aglutinación frente a algunas lectinas, reflejan que efectivamente se está dando una transformación hacia una forma infectiva. La generación de promastigotes infectivos dependiente de la fase de crecimiento se ha demostrado también en especies de Leishmania donovani, Leishmania major, Leishmania tropica, Leishmania amazonensis y Leishmania braziliensis $(7,8,11,12,14)$.

Este método de separación o purificación de formas metacíclicas por aglutinación con lectinas es un método restringido a cepas de L. major y dependiente de la expresión del glicoconjugado de superficie denominado lipofosfoglicano (LPG). Se han desarrollado protocolos similares para purificar promastigotes metacíclicos por medio de anticuerpos monoclonales específicos de LPG procíclico en $L$. tropica (13) y L. donovani (14). Recientemente, Spath y Beverly (15) diseñaron un método de separación de formas metacíclicas, independiente de LPG, el cual mostró buena eficacia y correlación con el método basado en aglutinación con PNA; sin embargo, su utilidad práctica en otras especies diferentes a $L$. major aún no se ha evaluado. La transformación hacia una forma más infectiva in vitro es evidente o está bien documentada en especies de Leishmania del Viejo Mundo; sin embargo, no parece existir una infectividad estrictamente dependiente de la fase de crecimiento en cepas de Leishmania del subgénero Viannia (16).

\section{Cambios morfológicos, genéticos y bioquímicos asociados con metaciclogénesis en Leishmania}

En un principio se creyó que existía una forma particular de promastigotes en el intestino del vector, la cual se había adaptado luego para sobrevivir en el hospedero vertebrado (17). Más recientemente, se observó que existen formas diferentes, tanto morfológica como bioquímicamente distinguibles en el vector (18). El primer paso en el desarrollo del parásito en el vector es la transformación de amastigote a promastigote después de 18 a 24 horas de la ingestión. Estos promastigotes sufren procesos de replicación y diferenciación pasando por varios estadios morfológicamente distinguibles. Al menos, cinco formas se han descrito en el vector durante el desarrollo del parásito Leishmania (subgénero Leishmania): promastigotes procíclicos, nectomónadas, haptomónadas, paramastigotes y, finalmente, promastigotes metacíclicos (19). Las formas metacíclicas tienden a ser más pequeñas, delgadas y con un flagelo más largo, presentan alta movilidad y migran hacia la parte anterior del tracto digestivo ubicándose a la altura de la probóscide $(16,19)$. Como promastigotes metacíclicos, están listos para ser inoculados en el hospedero mamífero ante una nueva ingestión de sangre por el vector $(7,19)$.

Además de los cambios morfológicos, este proceso de metaciclogénesis va acompañado de la síntesis de moléculas expresadas exclusiva o diferencialmente en el promastigote infectivo 0 metacíclico $(20,21)$. Las moléculas mejor 
estudiadas, caracterizadas y asociadas con la metaciclogénesis son el LPG $(22,23)$ y la glicoproteína gp63 $(24,25)$, expresadas abundantemente en la superficie de los promastigotes, principalmente en cepas del subgénero Leishmania, pero que sufren una considerable disminución o están ausentes, según la especie, en el estadio de amastigote (23). En el subgénero Leishmania, el LPG se expresa sobre toda la superficie del promastigote, incluido el flagelo, y está organizado como un filamento densamente empaquetado que forma un glicocálix. Estructuralmente, el LPG es una molécula dividida en cuatro dominios: un lípido de anclaje tipo liso-1-o-alquilfosfatidilinositol, un núcleo hexacárido, una unidad repetitiva

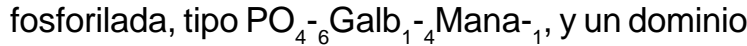
terminal, conformado en la mayoría de las especies caracterizadas por un disacárido o trisacárido (figura 1a) (32-34).

Las modificaciones del LPG durante la metaciclogénesis en las especies hasta ahora caracterizadas consisten básicamente en dos tipos de cambios: 1) incremento en el tamaño del LPG y 2) sustituciones o modificaciones en la composición de azúcares que conforman algunas de estas unidades repetitivas o del dominio terminal $(21,38)$. Se ha demostrado que el aumento en el tamaño del LPG se debe a una duplicación en el número de unidades repetitivas a medida que los promastigotes se diferencian desde fase de crecimiento logarítmica a estacionaria (figura 1b) $(35,36)$. Este cambio en el tamaño o grosor del glicocálix se ha observado por microscopía electrónica en promastigotes de L. donovani (37). En contraste, poco se conoce sobre la estructura y expresión de este glicoconjugado en Leishmania del Nuevo Mundo clasificadas dentro del subgénero Viannia, aunque parece que su expresión se encuentra bastante disminuida. Experimentos que empleaban una cepa de $L$. brazilienzis mostraron que esta cepa expresa 10 veces menos LPG que lo que expresa $L$. major (26).

La otra molécula ampliamente estudiada, la glicoproteína gp63, es una metaloproteasa y de

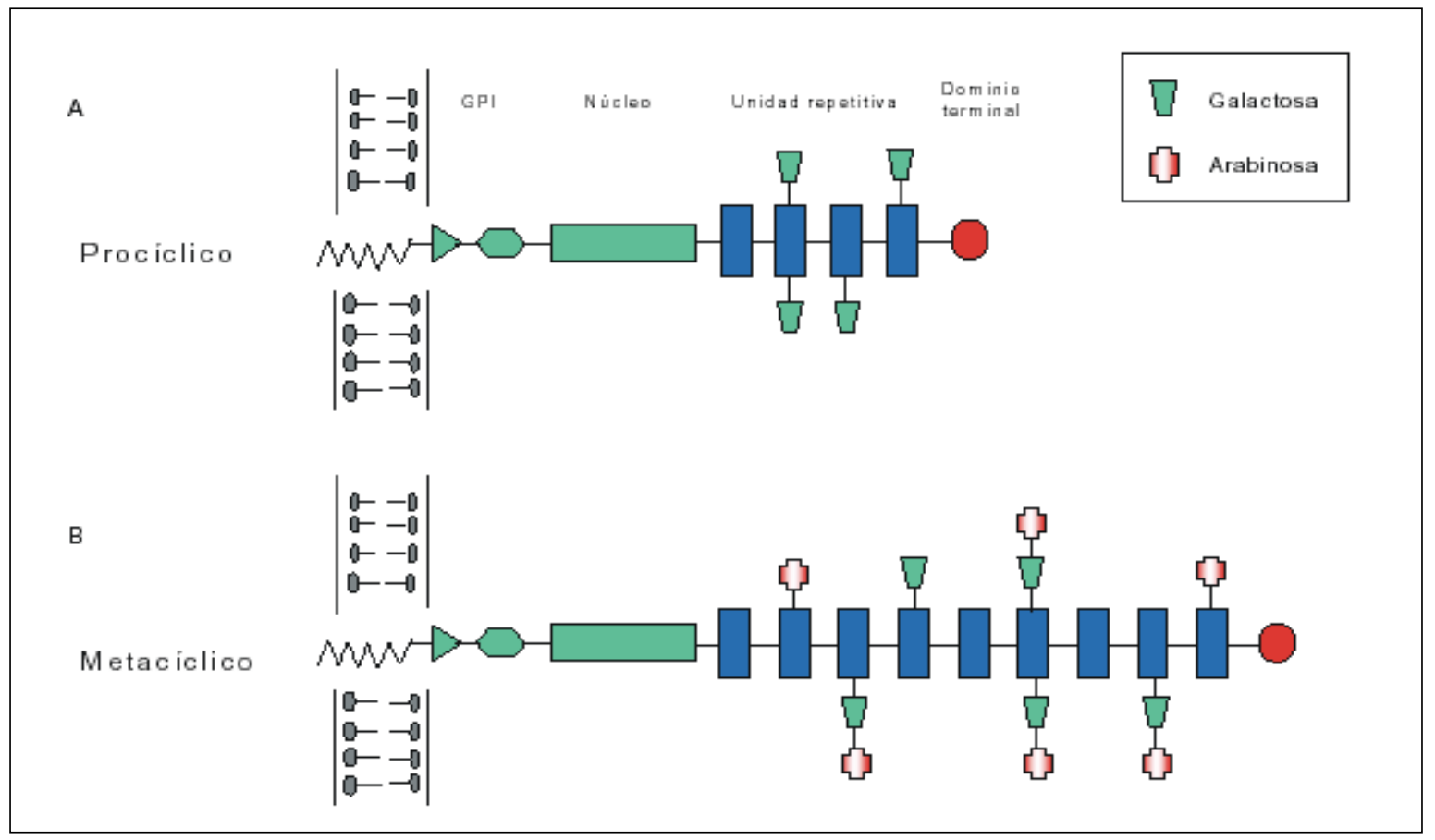

Figura 1. Modificaciones del LPG durante el proceso de metaciclogénesis: A. estructura del LPG en promastigotes procíclicos; B. estructura del LPG en promastigotes metacíclicos. 
la cual se sabe que presenta polimorfismo entre especies $(24,25)$ y cuya expresión se encuentra disminuida en los amastigotes (39-42). Se ha informado que el incremento en la infectividad observada en los promastigotes metacíclicos está asociado con un incremento en la expresión de gp63 (24). En contraste, en 1987, King y Chang (43) sugirieron que el aumento en la virulencia asociado a gp63 está mas correlacionado con el aumento en el grado de glicosilación, lo cual la hace más estable. La importancia del LPG y gp63 en el ciclo de vida de Leishmania y su asociación con metaciclogénesis se discutirá más adelante.

Otras moléculas como una ARN polimerasa putativa, una ATPasa putativa y una proteína relacionada con la hsp70 se sobrexpresan en el promastigote metacíclico de L. major (6); sin embargo, poco se conoce sobre la expresión de estas moléculas en otras especies de Leishmania y de su asociación con la metaciclogénesis.

Más recientemente, empleando técnicas para detectar genes expresados diferencialmente, se han identificado nuevas moléculas que muestran patrones particulares de expresión y localización y que están sobrexpresados en los promastigotes metacíclicos (figura 2). Entre estos nuevos genes se encuentra el producto del gen $B(27)$, los genes meta-1 (28), mat-1 (29), SHERP (30) y HASP (31).
Sin embargo, la función de los productos de estos genes en el ciclo de vida de Leishmania no está aún bien determinada y es objeto de estudio.

Los genes meta-1 y mat-1 fueron inicialmente identificados en $L$. major, aunque meta- 1 también ha sido recientemente caracterizado, parcial o totalmente, en L. amazonensis, L. donovani (44), L. infantum, L. panamensis y L. braziliensis (45). El gen meta-1 se expresa en mayor cantidad en el promastigote metacíclico de L. major (28); sin embargo, la evaluación de la expresión de este gen en promastigotes de Leishmania Viannia recién aislados de hámsters en nuestro laboratorio y cosechados los días 2, 4, 6 y 8 de crecimiento, no muestran este particular patrón de expresión (datos sin publicar). En una primera aproximación para tratar de elucidar la función del gen meta-1 mediante delección homóloga, se encontró que este gen parece ser esencial para Leishmania, dado que las dobles mutantes no fueron viables (44). Posteriormente, con el empleo de parásitos transfectados en los cuales se sobrexpresó esta proteína, se encontró que estos parásitos producían lesiones de mayor tamaño que la cepa control en ratones Balb/c (44), lo que sugiere un papel en la virulencia de Leishmania.

A diferencia de meta-1, el ARN mensajero de mat1 se expresa exclusivamente en el promastigote

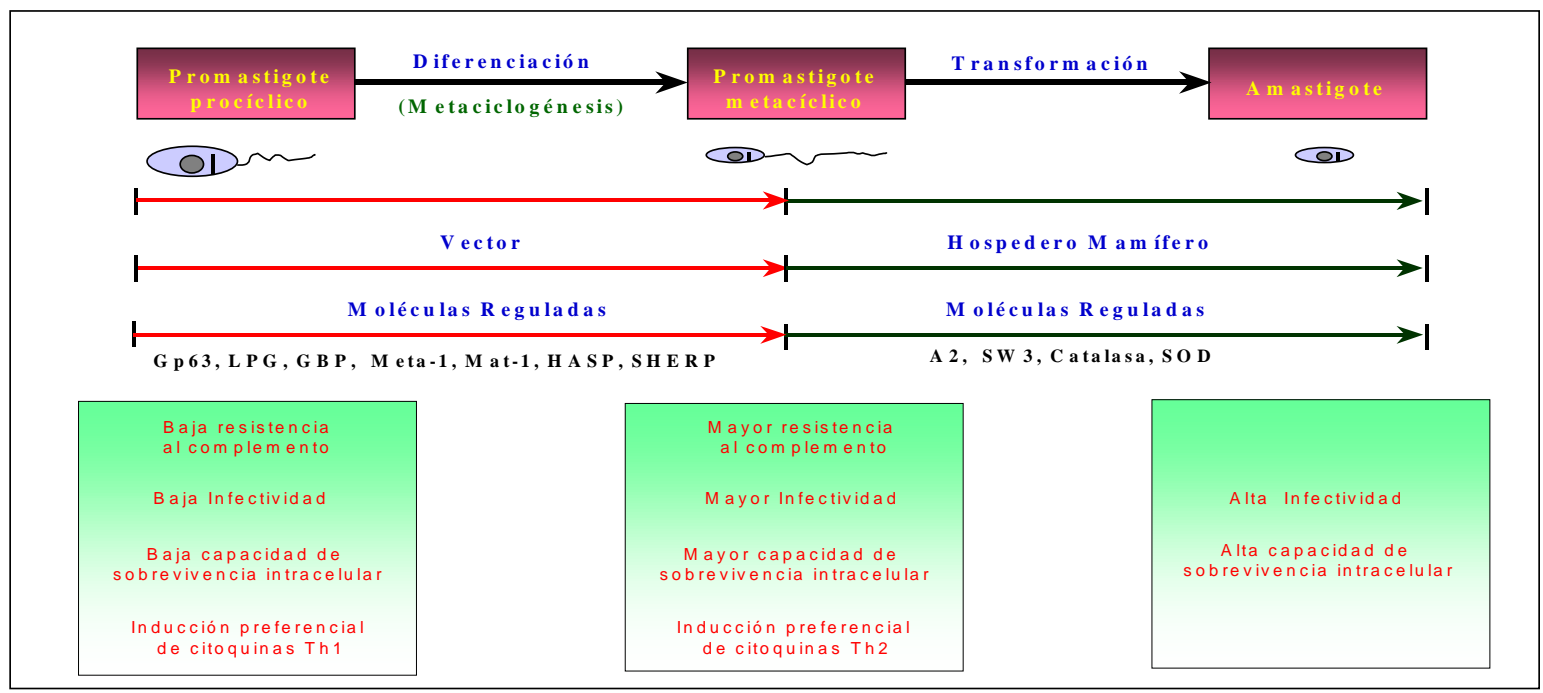

Figura 2. Diferenciación y transformación de los parásitos de Leishmania en el hospedero invertebrado y vertebrado, genes regulados en estos procesos y las implicaciones de la metaciclogénesis en el ciclo de vida de los promastigotes. 
metacíclico de L. major (29). En nuestro laboratorio, pudimos amplificar, clonar, secuenciar y expresar como proteína recombinante el marco de lectura del gen mat-1 de $L$. infantum (46). Sin embargo, no fue posible amplificar el marco de lectura de mat-1 en dos especies de Leishmania Viannia al emplear los cebadores diseñados a partir de las secuencias de L. majoro L. infantum. Adicionalmente, no fue posible detectar este gen en especies de Leishmania Viannia mediante análisis de Southern blot con el empleo de una sonda heteróloga obtenida de L. infantum (datos sin publicar). Estos resultados sugieren la presencia de un gen altamente variable entre especies del Viejo y Nuevo Mundo o que este gen no está presente en especies de Leishmania Viannia.

El producto del gen $B(48)$, las proteínas SHERP (30) y HASP $(27,31)$ son codificadas por genes localizados dentro del locus de la familia de genes denominada LmcDNA 16, que abarca una región de 15-16 Kb en L. major. Las evaluaciones serológicas con el empleo de la proteína o de péptidos recombinantes del gen B o HASP-B han detectado la presencia de anticuerpos en suero de personas infectadas con Leishmania, lo cual indica o sugiere que tales proteínas podrían estar jugando un papel en la inducción o modulación de la respuesta inmune del hospedero $(31,47)$. La proteína SHERP se ha encontrado a nivel del retículo endoplasmático y en la membrana externa de la mitocondria. Recientemente, infecciones realizadas con mutantes en ensayos de infección con macrófagos y supervivencia intracelular, tanto in vitro como in vivo, y obtenidas por deleción homóloga de la totalidad del locus LmcDNA 16, el cual contiene los genes SHERP y HASP, fueron igualmente virulentas a las cepas silvestres (48). Aunque la función de SHERP no ha sido establecida, se especula que éste podría ser un requerimiento específico para la función de algunos organelos durante el proceso de diferenciación de los promastigotes.

\section{Implicaciones de la metaciclogénesis en el ciclo de vida de Leishmania}

Para que el promastigote se transforme de promastigote procíclico a metacíclico, el parásito debe ser capaz de pasar varias barreras potenciales en el insecto vector, tales como: 1) las enzimas proteolíticas empleadas para digerir la sangre ingerida y 2) la membrana peritrófica, la cual es una barrera temprana para la migración del parásito hacia el epitelio intestinal. Pasar esta barrera, le permite al promastigote unirse al intestino del insecto vector para no ser barrido con la eliminación de la sangre digerida por el vector y transformarse en un promastigote metacíclico altamente infectivo y preadaptado para sobrevivir a los diferentes mecanismos microbicidas del sistema inmune del hospedero vertebrado.

Uno de los papeles atribuidos al LPG en el vector parece ser la potencial protección contra enzimas hidrolíticas. En 1986, Schlein y Romano (49) evidenciaron que las enzimas proteolíticas producidas durante la digestión de la sangre se inhibían por infección con L. major o por un factor excretado de L. major (presumiblemente, LPG). El papel protector del LPG contra las enzimas proteolíticas del vector se demostró posteriormente empleando mutantes de LPG (54), las cuales produjeron bajas tasas de infección a las 48 horas postingestión comparadas con la infección producida por la cepa silvestre (50); sin embargo, no existe una evidencia experimental directa de la inhibición de estas enzimas por el LPG. El otro papel del LPG en el vector parece ser en la adhesión de los parásitos al epitelio intestinal. La adhesión de los promastigotes al intestino del vector parece ser una propiedad inherente a los promastigotes procíclicos o no infectivos, propiedad que se pierde durante la transformación a promastigote metacíclico o infectivo, permitiendo, por consiguiente, la liberación y migración del parásito infectivo hacia la probóscide del insecto, para luego ser transmitido durante una nueva picadura. Se encontró que la unión y liberación del promastigote del epitelio intestinal está relacionada con modificaciones en los azúcares terminales del LPG (51) o con cambios conformacionales de la molécula (37). El papel del LPG en la unión de los promastigotes al intestino del vector se demostró empleando también mutantes (52) que no expresan LPG en 
su superficie. Con estas mutantes se observó que la capacidad de unión al intestino de su vector natural estaba muy disminuida cuando se comparaba con la cepa silvestre, la cual expresaba LPG en su superficie (53). Además, también se demostró que la unión de $L$. majoral tracto digestivo de Phlebotomus papatasi, su vector natural, puede ser completamente inhibida con LPG total o con unidades repetitivas purificadas de promastigotes procíclicos de esta misma especie (38).

Cuando los parásitos son inoculados en el hospedero mamífero, los promastigotes están expuestos transitoriamente a factores del complemento antes de penetrar a los macrófagos presentes en el sitio de la inoculación. Se sabe que los promastigotes procíclicos son más sensibles a la acción del complemento, aun en ausencia de anticuerpos anti-Leishmania. En contraste, los promastigotes metacíclicos son relativamente más resistentes o evaden mejor la acción del complemento (figura 2) $(54,55)$, aunque el grado de resistencia parece ser algo inherente a cada especie (55). Se han sugeridos varios mecanismos para explicar cómo los promastigotes evaden la acción del complemento: 1) una rápida invasión o incorporación a los macrófagos, acortando así el tiempo de exposición; 2) inactivación del complemento por componentes presentes en la saliva de los vectores, y 3) diferenciación del promastigote hacia un estadio infectivo o metacíclico que lo hace más resistente al complemento (55). EI LPG es una de las moléculas involucradas en la resistencia al complemento, dado que los experimentos que empleaban la cepa deficiente de LPG, denominada R2D2 (52), mostraron un mayor porcentaje de lisis en la cepa deficiente de LPG que en diferentes especies de Leishmania, normales para la expresión de esta molécula (56). Varias son las hipótesis que explican la resistencia de Leishmania al complemento mediada por LPG. Uno de estos mecanismos podría esta asociado con el incremento del grosor del glicocálix del LPG, el cual impide o bloquea la inserción del complejo de ataque a la membrana C5b-9, la membrana celular del promastigote (23) y es luego arrojado de la superficie del promastigote (57). Se cree que alguno de los mecanismos de resistencia al complemento elaborado por Leishmania es similar o ejerce su función de manera análoga a como lo hace el lipopolisacárido (LPS) bacteriano, el cual se ha demostrado que tiene efecto anticomplementario $(57,61)$.

Adicionalmente, se ha demostrado que los promastigotes metacíclicos tienen una mayor capacidad para transformar la fracción C3b del complemento a la forma inactiva C3bi o para fosforilar la fracción C3. La fracción C3 fosforilada no es hidrolizada en C3b y C3a, inhibiendo subsecuentemente las vías clásica y alterna del complemento. En la inactivación de la fracción C3b o la fosforilación de la fracción C3 se ha involucrado la glicoproteína gp63 $(54,58)$ y proteínas cinasas coincidencialmente sobrexpresadas en los promastigotes metacíclicos, respectivamente (59). Interesa anotar que la fracción inactiva C3bi del complemento es capaz de unirse luego a los promastigotes, presumiblemente a través de la misma gp63 o del LPG y ser empleada en la internalización o infección de los macrófagos por medio de los receptores del complemento $(60,62)$.

Una vez el promastigote invade el macrófago, éste es atacado por enzimas lisosómicas y por metabolitos reactivos del oxígeno. Nuevamente, la glicoproteína gp63 y el LPG parecen jugar un papel primordial en la supervivencia del parásito en el macrófago. La glicoproteína gp63, cuya actividad proteasa óptima se alcanza a $\mathrm{pH} 4$ como el que se encuentra en el fagolisosoma, está involucrada en la degradación inespecífica de enzimas lisosómicas deletéreas para el parásito (58). Por su parte, el LPG, un factor de virulencia multifuncional al menos en $L$. major, se ha implicado en varios procesos que conllevan a la desactivación del macrófago, como es la inhibición de la proteína cinasa C $(63,64)$, la cual está relacionada con la respuesta oxidativa del macrófago. Además, se ha visto que el LPG inhibe la fusión fagosoma-endosoma (65) y elimina eficientemente metabolitos tóxicos del oxígeno como el anión superóxido y radicales hidroxilos (23). La inhibición transitoria de la fusión fagosoma-endosoma puede entenderse como una estrategia del parásito para ganar tiempo hasta transformarse en amastigote, el cual es más resistente a 
enzimas hidrolíticas y al pH ácido encontrado en el fagolisosoma una vez ocurre la fusión fagosoma-endosoma. Sin embargo, el papel de LPG como factor de virulencia multifuncional en todas las especies de Leishmania fue puesto en duda recientemente, dado que los resultados de estudios que emplearon un mutante del gen lpg1 de $L$. major y $L$. mexicana mostraron resultados completamente contradictorios. Mientras que la mutación del gen Ipg 1 conlleva la pérdida de virulencia en $L$. major, no ocurre lo mismo cuando se muta este locus en $L$. mexicana, la cual infecta y sobrevive en líneas celulares de macrófagos y permanece infectiva para ratones $(66,67)$.

Dos tipos de respuesta inmune celular distinguibles por el patrón de citocinas y que afectan el resultado de la infección por Leishmania han sido bien caracterizados en el modelo murino con cepas de L. major. Una respuesta tipo 1 , la cual se asocia con resistencia a la infección y se caracteriza por la secreción de citocinas como IL-2, INF- $\gamma$ y IL-12, y una respuesta tipo 2, la cual se asocia con susceptibilidad a la enfermedad y en la que algunas de las citocinas secretadas son IL-4, IL10 y TGF- $\beta$ (68). Recientemente, se ha informado que los promastigotes de Leishmania modulan el patrón de citocinas y que esta regulación está influida por el estadio del promastigote (figura 2). Se ha encontrado que los promastigotes metacíclicos son pobres inductores de la expresión de IL-12 e IFN- $\gamma$ tanto en células mononucleares de sangre periférica como en monocitos humanos. En contraste, los promastigotes procíclicos obtenidos de la fase logarítmica de crecimiento mostraron una fuerte habilidad para inducir IL-12, INF- $\gamma$, TNF y IL-10 (69). Es probable que las moléculas sobrexpresadas en los promastigotes metacíclicos sean, quizás, las responsables de ejercer este control o de modular la expresión de un patrón particular de citocinas; sin embargo, no se han descrito evidencias directas en este aspecto.

La mayoría de trabajos que involucran metaciclogénesis se han realizado con cepas del Viejo Mundo, principalmente L. majory en menor proporción en $L$. donovani. Filogenética y clínicamente se han observado diferencias entre especies del subgénero Leishmania y subgénero Viannia; en consecuencia, no se conoce si muchos de estos fenómenos y mecanismos o la misma regulación en la expresión de algunos genes descritos en Leishmania del Viejo Mundo ocurren de manera similar o análoga en especies de Leishmania del Nuevo Mundo. Sin embargo, se ha demostrado que no todo lo que se encuentra o demuestra en una especie particular puede ser extrapolado directamente a todas las especies de Leishmania, como evidenciaron los experimentos con la mutación del gen lpg1, entre otros.

\section{Referencias}

1. Killick-Kendrick R. Biology of Leishmania in Phlebotomine sand flies. En: Lumsden WHR, Evans DA, editors. Biology of the Kinetoplastida. London: Academic Press; 1979. p.395-460.

2. Molyneux DH, Killick-Kendrick R. Morphology, ultrastructure and life cycles. En: Peters W, KillickKendrick R, editors. The leishmaniasis in biology and medicine. London: Academic Press; 1987. p.257.

3. Bandyopadhyay P, Ghosh DK, De A, Ghosh KN, Chaudhuri PP, Das P, et al. Metacyclogenesis of Leishmania spp.: species-specific in vitro transformation, complement resistance, and cell surface carbohydrate and protein profiles. Parasitology 1991;77:411-6.

4. Chares H, Matlashewski G. Developmental gene expression in Leishmania donovani: differential cloning and analysis of an amastigote-stage gene. Mol Cell Biol 1994;14:2975-84.

5. Langford CK, Burchmore RJ, Hart DT, Wagner W, Landfear SM. Biochemistry and molecular genetics of Leishmania glucose transporters. Parasitology 1994; 108:73-83.

6. Sacks DL. Metacyclogenesis in Leishmania promastigotes. Exp Parasitol 1989;69:100-3.

7. Sacks DL, Perkin PV. Identification of an infective stage of Leishmania promastigotes. Science 1984;223:14179.

8. Sacks DL, Perkin PV. Development of infective stage Leishmania promastigotes within phlebotomine sandflies. Am J Trop Med Hyg 1985;34:456-9.

9. Sacks DL, Hieny S, Sher A. Identification of cell surface carbohydrate and antigenic changes between noninfective and infective developmental stages of Leishmania major promastigotes. J Immunol 1985;135: 564-9.

10. da Silva R, Sacks DL. Metacyclogenesis is a major determinant of Leishmania promastigote virulence and attenuation. Infect Immun 1987;55:2802-6. 
11. Giannini MN. Effects of promastigotes growth phase, frequency of subcultures, and host age on promastigotes-initiated infections with Leishmania donovani in the golden hamster. J Protozool 1974;21: 521-7.

12. Wozencraft AO, Blackwell JM. Increased infectivity of stationary-phase promastigotes of Leishmania donovani: correlation with enhanced C3 binding capacity and CR3mediated attachment to host macrophages. Immunology 1987;60:559-63.

13. Lira R, Mendez S, Carrera L, Jaffe C, Neva F, Sack DL. Leishmania tropica: the identification and purification of metacyclic promastigotes and use in establishing mouse and hamster models of cutaneous and visceral disease. Exp Parasitol 1998;89:331-42.

14. Almeida MC, Cuba CA, de Sa CM, Pharoah MM, Howard KM, Miles MA. Metacyclogenesis of Leishmania (Viannia) braziliensis in vitro: evidence that lentil lectin is a marker of complement resistance and enhanced infectivity. Trans R Soc Trop Med Hyg 1993; 87:325-9.

15. Spath GF, Beverly SM. A lipophosphoglycanindependent method for isolation of infective Leishmania metacyclic promastigotes by density gradient centrifugation. Exp Parasitol 2001;99:97-103.

16. Rey JA, Travi BL, Valencia AZ, Saravia NG. Infectivity of the subspecies of the Leishmania braziliensis complex in vivo and in vitro. Am J Trop Med Hyg 1990;43:62331.

17. Adler S, Ber M. The transmission of Leishmania tropica by the bite of Phlebotomus papatasi. Indian J Med Res $1941 ; 29: 803-9$.

18. Killick-Kendrick R. The life cycle of Leishmania in the sandfly with special reference to the form infective to the vertebrade host. Ann Parasitol Hum et Comparee 1990;65:37-42.

19. Bates AP. The developmental biology of Leishmania promastigotes. Exp Parasitol 1994;79:215-8.

20. Coulson RM, Smith DF. Isolation of genes showing increased or unique expression in the infective promastigotes of Leishmania major. Mol Biochem Parasitol 1990;40:63-75.

21. Moody S. Molecular variation in Leishmania. Acta Tropica 1993;53:185-204.

22. Turco SJ. The lipophosphoglycan of Leishmania. Parasitol Today 1988;4:255-7.

23. Turco SJ, Descoteaux A. The lipophosphoglycan of Leishmania parasites. Annu Rev Microbiol 1992;46:6594.

24. Kweider M, Lemesre J, Darcy F, Kusnierz J, Capron A, Santoro F. Infectivity of Leishmania braziliensis promastigotes is dependent on the increasing expression of a 65,000-Dalton surface antigen. J Immunol 1987;138:299-305.
25. Davies C, Cooper A, Peacock A, Lane R, Blackwell J. Expression of $L P G$ and GP63 by different developmental stages of Leishmania major in the sandfly Phlebotomus papatasi. Parasitology 1990;101:337-43.

26. Muskus C, Segura I, Oddone R, Turco SJ, Leiby DA, Toro L, et al. Carbohydrate and LPG expression in Leishmania Viannia subgenus. J Parasitol 1997; 83:671-8.

27. Flinn HM, Rangarajan D, Smith DF. Expression of a hydrophilic surface protein in infective stages of Leishmania major. Mol Biochem Parasitol 1994;65:25970.

28. Nourbakhsh F, Uliana RB, Smith DF. Characterisation and expression of a stage-regulated gene of Leishmania major. Mol Biochem Parasitol 1996;76:201-13.

29. Brodin TN, Heath S, Sacks DL. Genes selectively expressed in the infectious (metacyclic) stage of Leishmania major promastigotes encode a potential basic-zipper structural motif. Mol Biochem Parasitol 1992;52:241-50.

30. Knuepfer E, Stierhof YD, McKean PG, Smith D. Characterisation of a differentially expressed protein that shows an unusual localization to intracellular membranes in Leishmania major. Biochem J 2001;356: 335-44.

31. Alce T, Gokool S, McGhie D, Stager S, Smith DF. Expression of hydrophilic surface proteins in infective stages of Leishmania donovani. Mol Biochem Parasitol 1999;102:191-6.

32. McConville M, Thomas T, Ferguson MA, Homans SW. Structure of the lipophosphoglycan from Leishmania major. J Biol Chem 1990;265:19611-23.

33. Ilg $\mathbf{T}$, Etges $\mathbf{R}$, Overath $\mathbf{P}$, McConville M, ThomasOates J, Thomas J, et al. Structure of Leishmania mexicana lipophosphoglycan. J Biol Chem 1992;267: 6834-40.

34. Thomas JR, McConville MJ, Thomas-Oates JE, Homans SW, Ferguson MA, Gorin PA, et al. Refined structure of lipophosphoglycan of Leishmania donovani. J Biol Chem 1992;267:6829-33.

35. McConville M, Turco SJ, Ferguson MA, Sacks DL. Developmental modification of lipophosphoglycan during the differentiation of Leishmania major promastigotes to an infectious stage. EMBO J 1992;11:3593-600.

36. Sacks DL, Brodin TN, Turco SJ. Developmental modification of the lipophosphoglycan from Leishmania major promastigotes during metacyclogenesis. Mol Biochem Parasitol 1990;42:225-32.

37. Sacks DL, Pimenta FP, McConville MJ, Schneider P, Turco SJ. Stage-specific binding of Leishmania donovani to the sandfly vector midgut is regulated by conformational changes in the abundant surface lipophosphoglycan. J Exp Med 1995;181:685-95. 
38. Sacks DL, Saraiva EM, Rowton E, Turco SJ, Pimenta PF. The role of the lipophosphoglycan of Leishmania in vector competence. Parasitology 1994;108:55-62.

39. Colomer-Gould V, Quintao LG, Keithly J, Nogueira N. A common major surface antigen on amastigotes and promastigotes of Leishmania species. J Exp Med 1985;162:902-16.

40. Chaudhuri G, Chaudhuri M, Pan A, Chang KP. Surface acid proteinase (gp63) of Leishmania mexicana. A metalloenzyme capable of protecting liposomeencapsulated proteins from phagolysosomal degradation by macrophages. J Biol Chem 1989;264: 7483-9.

41. Pimenta PF, Saraiva EBB, Sacks DL. The comparative fine structure and surface glycoconjugate expression of three life stages of Leishmania major. Exp Parasitol 1991;72:191-204.

42. Schneider P, Rosat JP, Bouvier J, Louis J, Bordier C. Leishmania major: differential regulation of the surface metalloprotease in amastigotes and promastigotes stages. Exp Parasitol 1992;75:196-206.

43. King JA, Chang KP. Biological and biochemical characterization of tunicamycin resistant Leishmania mexicana: mechanisms of drug resistance and virulence. Infect Immun 1987;55:1692-700.

44. Uliana RB, Neena G, Freymuller E, Smith FD. Leishmania: overexpression and comparative structural analysis of the stage-regulated meta-1 gene. Exp Parasitol 1999;92:183-91.

45. Berberich C, Marín M, Ramírez JR, Muskus C, Vélez ID. The metacyclic stage-expressed meta-1 gene is conserved between Old and New World Leishmania species. Mem Inst Osw Cruz 1988;93:819-21.

46. Marín M, Muskus C, Ramírez JR, Arbelaez LF, Alzate JF, Berberich $\mathbf{C}$. The gene encoding the metacyclogenesis-associated transcript mat- 1 is conserved in the genus Leishmania and shows a tendency to form dimers upon protein expression. Parasitol Res 2000;86:431-5.

47. Jensen AT, Gaafar A, Ismail A, Christensen CB, Kemp M, Hassan AM. Serodiagnosis of cutaneous leishmaniasis: assessment of an enzyme-linked immunosorbent assay using a peptide sequence from gene B protein. Am J Trop Med Hyg 1996;55:490-5.

48. McKean PG, Denny PW, Knuepfer E, Keen JK, DF smith. Phenotypic changes associated with deletion and overexpression of stage-regulated gene family in Leishmania. Cell Microbiol 2001;3:511-23.

49. Schlein Y, Romano H. Leishmania major and Leishmania donovani: effects of proteolytic enzymes of Phlebotomus papatasi (Diptera, Psychodidae). Exp Parasitol 1986;62:376-80.

50. Borovsky D, Schlein Y. Trypsin and chymotrypsin-like enzymes of the sandfly Phlebotomus papatasi infected with Leishmania and their possible role in vector competence. Med Vet Entom 1987;1:235-42.

51. Pimenta PF, Turco SJ, McConville M, Lawyer P, Perkins P, Sacks DL. Stage-specific adhesion of Leishmania promastigotes to the sand fly midgut. Science 1992;256:1812-5.

52. Ryan AK, Garraway LA, Descoteaux A, Turco SJ, Beverley SM. Isolation of virulence genes directing surface glycosyl-phosphatidylinositol synthesis by functional complementation. Proc Natl Acad Sci USA 1993;90:8609-13.

53. Pimenta PF, Saraiva MB, Rowton E, Modi GB, Garraway LA, Beverley SM, et al. Evidence that the vectorial competence of phlebotomine sand flies for different species of Leishmania is controlled by structural polymorphisms in the surface lipophosphoglycan. Proc Natl Acad Sci USA 1994;91:9155-9.

54. Bogdan $\mathbf{C}$, Rollinghoff $\mathbf{M}$. The immune response to Leishmania: mechanisms of parasite control and evasion. Int J Parasitology 1998;28:121-34.

55. Franke ED, McGreevy PB, Katz SP, Sack DL. Growth cycle-dependent generation of complement-resistant Leishmania promastigotes. J Immunol 1985;134:2713-8.

56. Muskus C. Papel del LPG y carbohidratos en la virulencia de Leishmania viannia (tesis). Cali, Colombia: Universidad del Valle; 1997.

57. Puentes SM, da Silva RP, Sacks DL, Hammer CH, Joiner KA. Serum resistance of metacyclic stage Leishmania major promastigotes is due to release of $\mathrm{C} 5 \mathrm{~b}$ 9. J Immunol 1990;145:4311-6.

58. Chaudhuri G, Chang KP. Acid protease activity of major surface membrane glycoprotein (gp63) from Leishmania mexicana promastigotes. Mol Biochem Parasitol 1988;27:43-52.

59. Hermoso T, Fishelson Z, Becker S, Hirschberg K, Jaffe CL. Leishmanial protein kinases phosphorylate components of the complement cascade. EMBO J 1991 ; 10:4061-7.

60. Rusell DG, Wright SD. Complement receptor type 3 (CR3) binds to an Arg-Gly-Asp containing region of the major surface glycoprotein, gp63 of Leishmania promastigotes. J Exp Med 1988;168:279-92.

61. Brittingham A, Morrison CJ, McMaster WR, McGwire BS, Chang KP, Mosser DM. Role of the Leishmania surface protease gp63 in complement fixation, cell adhesion and resistance to complement-mediated lysis. $\mathrm{J}$ Immunol 1995;155:3102-11.

62. Joiner KA, Grossman N, Schmetz M, Leive L. C3 binds preferentially to long chain lipopolysaccharide during alternative pathway activation by Salmonella montevideo. J Immunol 1986;136:710-5.

63. McNeely TB, Rosen G, Londner MV, Turco SJ. Inhibitory effects on protein kinase $C$ by lipophos- 
phoglycan and glycosyl-phosphatidylinositol antigens of Leishmania. Biochem J 1989;259:601-4.

64. Giorgione JR, Turco SJ, Epand RM. Transbilayer inhibition of protein kinase $C$ by the lipophosphoglycan from Leishmania donovani. Proc Natl Acad Sci USA 1996;93:11634-9.

65. Desjardins M, Descoteaux A. Inhibition of phagolysosomal biogenesis by the Leishmania lipophosphoglycan. J Exp Med 1997;185:2061-8.

66. Ilg T. Lipophosphoglycan is not required for detection of macrophages or mice by Leishmania mexicana. EMBO J 2000;19:1953-62.
67. Turco SJ, Spath GF, Beverly SM. Is lipophosphoglycan a virunce factor? A surprising diversity between Leishmania species. Trends in Parasitology 2001;17: 223-6.

68. Reiner SL, Locksley RM. The regulation of immunity to Leishmania major. Annu Rev Immunol 1995;13:51-77.

69. Sartori A, Oliveira M, Scott P, Trinchieri G. Metacyclogenesis modulates the ability of Leishmania promastigotes to induce IL-12 production in human mononuclear cells. J Immunol 1997;159:2849-57. 